\title{
Uso do recurso Stories para avaliação do conhecimento dos usuários do Instagram a respeito de PANCs, com destaque para a ora-pro-nóbis
}

\author{
Evaluation of the knowledge about ora-pro-nóbis from the perspective of the use of the stories tool \\ in the social network Instagram
}

Evaluación del conocimiento de ora-pro-nóbis desde la perspectiva del uso de la herramienta de

stories en la red social Instagram

Recebido: 16/06/2021 | Revisado: 22/06/2021 | Aceito: 24/06/2021 | Publicado: 10/07/2021

\author{
Thiago Vieira de Moraes \\ ORCID: https://orcid.org/0000-0003-1740-9884 \\ Universidade Federal do Estado do Rio de Janeiro, Brasil \\ E-mail: biomedicothiagovieira@yahoo.com.br \\ Joao Paulo Gonçalves Ferreira \\ ORCID: https://orcid.org/0000-0002-7703-6605 \\ Universidade Federal do Estado do Rio de Janeiro, Brasil \\ E-mail: jpferreira.jpg@gmail.com \\ Kelly Gloria do Nascimento Oliveira \\ ORCID: https://orcid.org/0000-0001-5359-1711 \\ Universidade do Estado do Rio de Janeiro, Brasil \\ E-mail: kellygno@yahoo.com.br
}

\begin{abstract}
Resumo
O conhecimento sobre a diversidade da flora brasileira com potencial alimentício, com destaque para as Plantas Alimentícias Não Convencionais (PANCs), é importante para ampliarmos as opções de alimentos saudáveis para uma população em contínuo crescimento. Dentre as PANCs nativas do Brasil, a ora-pro-nóbis (OPN) vem ganhando espaço na pesquisa científica e no mercado consumidor por seu potencial nutritivo e funcional. Em função do seu alcance público, o uso de redes sociais como o Instagram é interessante para avaliar mercados, divulgar, popularizar e esclarecer dúvidas, por exemplo, com relação à segurança do consumo desses novos produtos. Este estudo utilizou a ferramenta Stories com o objetivo de avaliar o conhecimento dos usuários do Instagram sobre PANCs e, mais especificamente, sobre OPN. Um total de 10 perguntas (objetivas e discursivas) foi disponibilizado no Instagram Stories ao longo de 24 horas. Os dados foram analisados de acordo com o engajamento do público e com relação à frequência simples e relativa das respostas. Os maiores engajamentos obtidos foram na pergunta sobre consumo $(27,20 \%)$ e conhecimento $(26,67 \%)$ de OPN. A maior frequência de respostas positivas $(87,56 \%)$ foi obtida na pergunta que questionava se o indivíduo consumiria produtos derivados de OPN e a maior frequência de respostas negativas $(84,81 \%)$ foi obtida na pergunta sobre a utilização de PANC na alimentação. O público alcançado demonstrou conhecimento e experiência no consumo de folhas de OPN, sendo que seu potencial proteico foi considerado a característica mais atrativa desse tipo de alimento. Apesar da pouca utilização de PANCs na alimentação, o público consultado mostrou interesse em consumir a farinha e outros produtos de OPN.
\end{abstract}

Palavras-chave: Ora-pro-nóbis; Instagram; Questionário; Comunicação científica.

\begin{abstract}
The knowledge on the diversity of Brazilian flora and its nutritional potential, especially regarding Unconventional Food Plants (UFPs), is important in order to expand the options of healthy foods for a continuously growing population. Among the UFPs native to Brazil, ora-pro-nóbis (OPN) has been gaining ground in scientific research and in the consumer market due to its nutritional and functional potential. Givento its public reach, the use of social networks such as Instagram is relevant for assessing markets, publicizing, popularizing and elucidating. For instance, regarding the safety of in consuming new products. This study used the Instagram Stories tool to assess Instagram users' knowledge on UFPs and, more specifically, on OPN. A total of 10 questions (objective and discursive) were posted on Instagram Stories over the course of 24 hours. Data was analyzed according to audience engagement and in relation to the simple and relative frequency of responses. The highest engagements obtained were in the question about consumption $(27.20 \%)$ and knowledge $(26.67 \%)$ of OPN. The highest frequency of positive answers $(87.56 \%)$ was obtained in the question that asked whether the individual would consume OPN-derived products and the highest frequency of negative answers $(84.81 \%)$ was obtained in the question about the use of UFP in food. The reached audience demonstrated knowledge and previous experience in consuming OPN leaves, and its protein potential was considered the most attractive feature of this type of food. Despite the little use of UFPs in food, the consulted public showed interest in consuming flour and other OPN-derived products.
\end{abstract}


Keywords: Ora-pro-nóbis; Instagram; Questionnaire; Scientific communication.

\section{Resumen}

El conocimiento sobre la diversidad de la flora brasileña con potencial nutricional, en especial las Plantas Alimentarias No Convencionales (PANC), es importante para ampliar las opciones de alimentos saludables para una población en continuo crecimiento. Entre las PANC originarias de Brasil, ora-pro-nóbis (OPN) ha ganado terreno en la investigación científica y en el mercado de consumo por su potencial nutricional y funcional. Por su alcance público, el uso de redes sociales como Instagram es interesante para evaluar mercados, dar a conocer, popularizar y aclarar dudas, por ejemplo, en cuanto a la seguridad del consumo de estos nuevos productos. Este estudio utilizó la herramienta Stories para evaluar el conocimiento de los usuarios de Instagram acerca de las PANC, específicamente la OPN. Se publicaron un total de 10 preguntas (objetivas y discursivas) en Instagram Stories durante 24 horas. Los datos fueron analizados según la participación de la audiencia y en relación con la frecuencia simple y relativa de las respuestas. Los mayores compromisos obtenidos fueron en la pregunta sobre el consumo $(27,20 \%)$ y el conocimiento $(26,67 \%)$ de OPN. La mayor frecuencia de respuestas positivas $(87,56 \%)$ se obtuvo en la pregunta en que se cuestiona si el individuo consumiría productos derivados de OPN y la mayor frecuencia de respuestas negativas $(84,81 \%)$ se obtuvo en la pregunta sobre el uso de PANC en los alimentos. La audiencia alcanzó conocimiento y experiencia demostrados en el consumo de hojas de OPN, y su potencial proteico fue considerado el rasgo más atractivo de este tipo de alimentos. A pesar del escaso uso de PANC en alimentos, el público consultado mostró interés en consumir harina y otros productos OPN.

Palabras clave: Ora-pro-nóbis; Instagram; Cuestionario; Comunicación científica.

\section{Introdução}

O Brasil possui $13 \%$ da riqueza da biota mundial. Só no reino vegetal são reconhecidas cerca de 35 mil espécies, sendo a maioria (55\%) endêmica (Stehmann \& Sobral, 2017). Naturalmente, como observado por Valente et al. (2020), a distribuição dessa riqueza pelo território se dá de maneira heterogênea pelos biomas. Entretanto, essa heterogeneidade não se reflete na base da alimentação dos brasileiros, que é muito semelhante, independente da região.

Acredita-se que pelo menos $10 \%$ da flora brasileira possui partes comestíveis que poderiam ser exploradas para incrementar a diversificação dietética (Kinupp \& Lorenzi, 2014). Além das espécies nativas, há um grande número de espécies exóticas naturalizadas com similar potencial alimentício. Essas plantas, nativas ou exóticas, por serem subutilizadas ou negligenciadas, de baixa ou nenhuma comercialização, recebem o título de Plantas Alimentícias Não Convencionais (PANCs) (Kinupp \& Lorenzi, 2014; Valente et al., 2020).

Nos últimos anos as PANCs vêm ganhando espaço nas discussões acadêmicas, nas mídias, feiras de comércio, e principalmente na mesa da população (Borges, 2017). Como observado por Junqueira \& Perline (2019), sob uma lente mais antropológica, elas vêm sendo resignificadas de "mato" para alimento, de "comida de pobre" para alimento gourmet, ocupando uma nova posição nas práticas sociais e contribuindo para a revalorização de práticas e culturas contra-hegemônicas. Além disso, Valente et al. (2020) também ressalta a importância desse resgate e apropriação dos conhecimentos sobre PANCs pelos pequenos e médios agricultores. Tendo em vista que a agricultura familiar detém cerca de $20 \%$ das terras e responde por aproximadamente $38 \%$ da produção nacional (Castro, 2015), o cultivo dessas plantas poderia representar um fortalecimento econômico e social dessa parcela produtiva.

A Pereskia aculeata Miller, mais conhecida como ora-pro-nóbis (OPN), é uma PANC da família das cactáceas que vem cada vez mais ganhando visibilidade por conta de sua interessante composição nutricional e propriedades bioativas. Seu potencial proteico, de fibras e minerais, bem como seu conteúdo de compostos do metabolismo secundário com propriedades anti-inflamatórias, antioxidantes e cicatrizantes tem contribuído para essa popularização da ora-pro-nóbis na mesa dos brasileiros (Almeida et al., 2014; Moraes et al., 2020; Pinto et al., 2016; Souza et al., 2016).

Apesar dessa crescente popularização da Ora-pro-nóbis e de outras PANCs, alguns consumidores ainda relutam em consumir esse tipo de produto por conta de dúvidas com relação a sua toxicidade e, também, por uma questão de hábito alimentar. É necessário promover ações, envolvendo tanto os produtores quanto os consumidores, a fim de incentivar a 
produção e consumo dessas plantas (Valente et al., 2020). Uma rápida busca bibliográfica por bases de dados como o "Google Acadêmico" ou o "Periódicos CAPES" já é capaz de mostrar que inúmeras ações voltadas para a popularização das PANCs vêm sendo tomadas por diversos grupos de estudo nos últimos anos (Moraes et al., 2018). No entanto, pelo menos no âmbito acadêmico, a utilização de redes sociais (como o Instagram) para esse fim ainda é relatada de forma escassa, porém de suma importância para disseminação do conhecimento (Costa, 2019; Moraes et al., 2021; Gomes et al., 2021).

Com mais de 90 milhões de usuários no Brasil (Statista, 2021), o Instagram é considerado a rede social mais utilizada pela população, inclusive para fins comerciais e de divulgação de informações. Há incontáveis perfis que geram ou compartilham conteúdo sobre PANCs e que têm uma significativa visibilidade. Isso reflete o crescente interesse na busca por melhores hábitos alimentares, abrindo espaço para a divulgação de informações cientificamente válidas e para a coleta de dados sobre a percepção do público em relação a essas plantas.

Nesse contexto, tendo em vista a importância da avaliação e da disseminação do conhecimento sobre PANCs e conhecendo o grande alcance que essas redes sociais possuem, o objetivo deste estudo foi avaliar o conhecimento de indivíduos registrados no Instagram sobre questões gerais relacionadas às PANCs, em especial à P. aculeata Miller (ora-pronóbis).

\section{Metodologia}

Para o desenvolvimento deste trabalho utilizou-se uma abordagem qualitativa, na qual os dados foram obtidos através de um questionário que foi aplicado aos usuários cadastrados na plataforma Instagram. Este estudo é considerado exploratório, com recorte transversal, sendo capaz de fornecer aos autores informações sobre o conhecimento e a utilização de PANCs por parte de uma parcela dos usuários dessa rede social (Estrela, 2018).

Após o login no próprio perfil do autor (@ thiagovieiramoraes) na rede social, no dia 19 de fevereiro de 2021, os visitantes tiveram acesso às perguntas que ficaram disponíveis por um período de 24 horas. O recurso stories possibilitou, então, a quantificação da interação do conteúdo postado com os seis mil seguidores desta conta (David et al., 2019). Foram disponibilizadas perguntas fechadas (7), pergunta aberta (1) e questões de múltipla escolha (2). O questionário utilizado no presente estudo foi adaptado do trabalho desenvolvido por Gralha (2020), que discute o nível de conhecimento dos agricultores familiares em relação às plantas alimentícias não convencionais. Para fins estatísticos, a análise dos dados foi realizada através de uma abordagem percentil descritiva, com base nas frequências absolutas e relativas das respostas.

\section{Resultados e Discussão}

$\mathrm{Na}$ Tabela 1 estão dispostas as perguntas na ordem em que foram exibidas para o público através do visualizador do stories de forma monádica, ou seja, um questionamento por vez. Logo após o participante contribuir com sua resposta, os resultados parciais de cada pergunta lhe eram exibidos em porcentagens. O número de participantes de cada pergunta foi calculado a partir da soma das respostas válidas computadas.

O engajamento a cada pergunta foi calculado como a razão entre o número de respostas válidas obtidas e o número de visualizações, multiplicada por 100. O número de visualizações diferiu entre as perguntas, assim como o número de respostas válidas. Foi possível observar um decréscimo no número de visualizações das perguntas à medida que os participantes avançavam no questionário. As exceções foram as perguntas nove e dez, que apresentaram um número de visualizações superior à pergunta oito. Isso pode ser explicado pelo fato de que os participantes foram instruídos a pularem a pergunta oito, caso a resposta à pergunta sete fosse negativa.

As perguntas de número seis, sete e dez, que citaram a OPN enquanto PANC obtiveram valores superiores a 20\% de engajamento. Isso pode ser atribuído à crescente popularização desta planta tanto no meio acadêmico, quanto no não 
acadêmico. $\mathrm{O}$ crescente número de publicações sobre OPN nos últimos anos, bem como sua forte presença na região sudeste, são fatores que contribuem para a popularização desta PANC. Por exemplo, a ocorrência anual do Festival de Ora-Pró-Nóbis em Sabará (MG), um evento gastronômico que contribui para a manutenção turística da cidade, ressalta a relevância cultural e econômica desta planta, bem como contribui para a disseminação de conhecimentos e incentiva o uso desta planta pela população.

As perguntas seis e dez foram as que apresentaram as maiores taxas de respostas positivas, ou seja, a opção pelo sim foi preferencialmente selecionada pelo público para responder a essas duas perguntas: 60,06\% das pessoas afirmaram que já conheciam a ora-pró-nóbis enquanto PANC (pergunta seis), e 87,56\% afirmaram que consumiriam a farinha ou outros derivados desta planta (pergunta dez). Isso indica o potencial comercial da ora-pró-nóbis e ressalta a importância da divulgação de informações confiáveis sobre sua composição e propriedades funcionais, o que poderia estimular ainda mais o consumo de produtos derivados ou enriquecidos com esta planta por consumidores em potencial.

Já as perguntas de número um, dois e três apresentaram os maiores percentuais de respostas negativas: 74,16\% apontaram não conhecer plantas espontâneas (daninhas, invasoras) que podem ser utilizadas na alimentação (pergunta um); 84,81\% afirmaram não utilizar essas plantas na alimentação (pergunta dois); 84,51\% afirmaram nunca terem notado a comercialização dessas plantas (pergunta três). Esse alto percentual de respostas negativas pode ser explicado pelo possível desconhecimento dos termos "planta espontânea", "daninha", "invasora" por parte do público consultado e, também, pela não associação desses termos com o acrônimo PANC.

Na questão cinco, sobre quais partes das PANCs poderiam ser consumidas, a opção "todas as alternativas" foi a mais escolhida $(75,34 \%)$, seguida das folhas $(11,99 \%)$, da flor $(10,27 \%)$ e do caule $(2,40 \%)$.

A pergunta de número oito era a única de resposta aberta, onde os participantes deveriam responder com suas palavras sobre quais partes da ora-pro-nóbis já haviam consumido, caso o consumo já tivesse ocorrido. As folhas da espécie foram a parte da planta mais citada (91,23\%), seguida de frutos e talos (3,51\% cada) e, por fim, as flores $(1,75 \%)$.

A utilização da farinha de ora-pró-nóbis para o enriquecimento e substituição da farinha de trigo em pães, massa para macarrão e bolos já tem sido explorada por alguns autores com resultados que mostram bons índices de aceitação em análises sensoriais (Arruda et al., 2016; Martinevski et al., 2013; Oliveira et al., 2008; Rocha et al., 2009; Santo Manoel, 2019). Além disso, Moraes et al. (2020) avaliaram o potencial antioxidante e o conteúdo de compostos fenólicos de chás elaborados com a farinha do caule da ora-pró-nóbis e mostraram que o chá dessa parte da planta pode ser uma fonte interessante de compostos fenólicos com um potencial antioxidante moderado.

Ainda sobre a pergunta de número oito, o menor número de visualizações e engajamento relativo observados podem estar relacionados aos diferentes modos de entrada da resposta. Ao contrário das demais perguntas, onde foram apresentadas alternativas que poderiam ser marcadas, nesta pergunta havia um campo de texto, o qual demandaria do participante mais tempo para a realização da atividade.

Na pergunta nove, quando questionados sobre qual seria o principal benefício associado ao consumo de ora-pró-nóbis, os participantes elencaram o potencial proteico com maior frequência $(44,04 \%)$, seguido do seu potencial antioxidante $(38,86 \%)$; nenhum dos citados $(10,36 \%)$ e efeito cicatrizante $(6,74 \%)$. Isto demonstra que a espécie OPN é vista como uma fonte proteica pela população.

A pergunta dez investiga a intenção de compra dos participantes de produtos derivados da OPN. A maior parte $(87,56 \%)$ dos 225 indivíduos que responderam a essa pergunta indicaram interesse em consumir a farinha e outros produtos derivados da ora-pró-nóbis. Isso estimula a realização de novas pesquisas para o desenvolvimento de tecnologias que viabilizem a formulação de produtos relacionados a esse tipo de matriz (Pereskia aculeata Miller). 
Research, Society and Development, v. 10, n. 8, e17910817226, 2021

(CC BY 4.0) | ISSN 2525-3409 | DOI: http://dx.doi.org/10.33448/rsd-v10i8.17226

Tabela 1. Perguntas do questionário, visualizações, engajamento e frequência percentual de respostas.

\begin{tabular}{|c|c|c|c|c|c|c|c|c|c|c|c|c|c|c|}
\hline $\mathrm{N}^{\mathrm{o}}$ & Pergunta & Visualizações & $\mathrm{N}$ & Engajamento \% & $\operatorname{Sim} \%$ & Não \% & \multicolumn{4}{|c|}{ Respostas múltipla escolha \% } & \multicolumn{4}{|c|}{ Respostas de pergunta aberta $\%$} \\
\hline 1 & $\begin{array}{l}\text { Conhece ou ouviu falar de } \\
\text { alguma "planta espontânea" } \\
\text { (daninha, invasora) que pode } \\
\text { ser utilizada na alimentação? }\end{array}$ & 1778 & 329 & 18,50 & 25,84 & 74,16 & & & & & & & & \\
\hline 2 & $\begin{array}{l}\text { Você utiliza ou utilizou } \\
\text { destas plantas na } \\
\text { alimentação? }\end{array}$ & 1624 & 316 & 19,46 & 15,19 & 84,81 & & & & & & & & \\
\hline 3 & $\begin{array}{l}\text { Você já viu no comércio a } \\
\text { venda destas plantas? }\end{array}$ & 1491 & 297 & 19,92 & 15,49 & 84,51 & & & & & & & & \\
\hline 4 & $\begin{array}{l}\text { Você conhece o termo } \\
\text { PANCs (Plantas Alimentícias } \\
\text { Não Convencionais)? }\end{array}$ & 1380 & 311 & 22,54 & 47,27 & 52,73 & & & & & & & & \\
\hline 5 & $\begin{array}{l}\text { Quais partes destas PANCs } \\
\text { podem ser consumidas? }\end{array}$ & 1312 & 292 & 22,26 & & & $\frac{\text { Folha }}{11,99}$ & $\begin{array}{l}\text { Flor } \\
10,27\end{array}$ & $\frac{\text { Caule }}{2,40}$ & $\begin{array}{l}\text { Todas as } \\
\text { alternativas } \\
75,34\end{array}$ & & & & \\
\hline 6 & Você conhece ora-pro-nóbis? & 1211 & 323 & 26,67 & 60,06 & 39,94 & & & & & & & & \\
\hline 7 & Já consumiu ora-pro-nóbis? & 1180 & 321 & 27,20 & 39,56 & 60,44 & & & & & & & & \\
\hline 8 & Se sim qual parte? Caso & 1004 & 57 & 5.68 & & & & & & & Folhas & Flores & Talos & Frutos \\
\hline & negativo, pule. & & & & & & & & & & 91,23 & 1,75 & 3,51 & 3,51 \\
\hline 9 & $\begin{array}{l}\text { Qual o principal benefício do } \\
\text { seu consumo? }\end{array}$ & 1123 & 193 & 17,19 & & & $\begin{array}{l}\text { Potencial } \\
\text { Antioxidante } \\
38,86\end{array}$ & $\begin{array}{l}\text { Potencial } \\
\text { Proteico } \\
44,04\end{array}$ & $\begin{array}{l}\text { Potencial } \\
\text { cicatrizante } \\
6,74\end{array}$ & $\begin{array}{l}\text { Nenhum } \\
\text { dos citados }\end{array}$ & & & & \\
\hline 10 & $\begin{array}{l}\text { Você consumiria a farinha e } \\
\text { outros produtos derivados da } \\
\text { ora-pro-nóbis? }\end{array}$ & 1106 & 225 & 20,34 & 87,56 & 12,44 & & & & & & & & \\
\hline
\end{tabular}

N: número absoluto de participantes calculado a partir da soma de respostas válidas computadas. Fonte: Autores (2021). 


\section{Conclusão}

Foi possível observar que a maioria dos participantes não conseguiu associar os termos “daninha”, “invasora” e/ou "planta espontânea" com alimentos que pudessem contribuir com a qualidade de vida e a diversidade de nutrientes em suas refeições. O uso do termo PANC parece ser mais conhecido do que os demais termos citados anteriormente, porém é valido ressaltar que boa parcela dessa população carece de informações sobre esse tema.

No que se refere à ora-pro-nóbis, percebe-se que é uma das PANCs mais conhecidas, mas seu consumo ainda pode ser substancialmente ampliado. Nota-se que o potencial proteico é a característica mais estimulante para o consumo dessa planta. Deste modo, sugere-se que novos estudos sejam desenvolvidos pela comunidade científica sobre a Pereskia aculeata Miller, a fim de ampliar o conhecimento sobre essa espécie de planta alimentícia não convencional. Tais ações visam estimular o consumo de produtos derivados e/ou enriquecidos com esta planta pelos consumidores, aumentando a diversidade de alimentos em nossa dieta e promovendo o cultivo e fortalecimento da agricultura familiar em nosso país. Por fim, sugere-se também avaliar o uso de outras redes sociais para entender de forma mais ampla a contribuição destas ferramentas na formação do conhecimento científico da população.

\section{Agradecimentos}

Agradecemos o apoio da CAPES, CNPq, FAPERJ e UNIRIO.

\section{Referências}

Almeida, M. E. F. de, Junqueira, A. M. B., Simão, A. A., \& Corrêa, A. D. (2014). Chemical characterization of the non-conventional vegetable known as orapro-nobis. Bioscience Journal, 30(Supplement), 431-439.

Arruda, H. S., De Cássia Sevilha, A., Pereira, T., \& Ferreira De Almeida, M. E. (2016). Substituição parcial da farinha de trigo pelas farinhas de grão-de-bico e de ora-pro-nobis na elaboração de um pão. In Nutrição Brasil (Vol. 15, Issue 2). https://doi.org/10.33233/NB.V15I2.222

Borges, C. K. G. D. (2017). Plantas alimentícias não convencionais (PANC) - a divulgação científica das espécies na cidade de Manaus [Universidade do Estado do Amazonas]. http://repositorioinstitucional.uea.edu.br//handle/riuea/2425

Castro, C. N. de. (2015). Desafios da agricultura familiar: o caso da assistência técnica e extensão rural. In http://www.ipea.gov.br. Instituto de Pesquisa Econômica Aplicada (Ipea). http://repositorio.ipea.gov.br/handle/11058/6492

Costa, F. V. (2019). Uso do Instagram como ferramenta de estudo: análise de um perfil da área biológica. Research, Society and Development, 8(10), e238102360. http://dx.doi.org/10.33448/rsd-v8i10.1360

David, F. de F. dos S., Silva, A. B. A. da, Baldasso, G., Marculino, C. H. de S., Almeida, J. V. de, \& Soltau, S. B. (2019). Uma proposta de uso do Instagram em metodologia aplicável em disciplinas do Ensino Médio. Research, Society and Development, 8(4), e1684959. https://doi.org/10.33448/rsd-v8i4.959 Estrela, C. (2018). Metodologia Científica: Ciência, Ensino, Pesquisa. Editora Artes Médicas.

Gralha, T. S. (2020). As plantas alimentícias não convencionais (PANC) a partir do conhecimento da agricultura familiar no município de Rio Grande - RS, um estudo de caso [Universidade Federal de Pelotas]. http://repositorio.ufpel.edu.br:8080/handle/prefix/6586

Gomes, B. I. R., da Silva, I. H., Alcantara, M. V. B. M., Alves, J. A., de Souza, S. R., de Oliveira, L. A., Zanetti, C. H. G. (2021). Quintal da saúde: plantas medicinais na promoção do cuidado. Brazilian Journal of Development, 7(3), 32567-32542. http://dx.doi.org/10.34117/bjdv7n3-804

Junqueira, A. H., \& Perline, E. A. (2019). Gosto, ideologia e consumo alimentar: práticas e mudanças discursivas sobre plantas alimentícias não convencionais-panc Tasting, Ideology and Food Consumption: practices and discursive changes about non-conventional food plants. Cadernos de Linguagem e Sociedade, 20(2), 17-35. https://doi.org/10.26512/les.v20i2.21772

Kinupp, V. F., \& Lorenzi, H. J. (2014). Plantas Alimentícias Não Convencionais (PANC) no Brasil: guia de identificação, aspectos nutricionais e receitas ilustradas.

Martinevski, C. S., Oliveira, V. R., Rios, A. O., Flores, S. H., \& Venzke, J. G. (2013). Utilização de bertalha (anredera cordifolia (ten.) steenis) e ora-pro-nobis (pereskia aculeata mill.) na elaboração de pães. Alimentos e Nutrição Araraquara, 24(3), 272. http://200.145.71.150/seer/index.php/alimentos/article/view/267

Moraes, T. V. de, Souza, M. R. A. de, Rocha, C. B, Moreira, R. F. A. (2018). Composição química da espécie Pereskia aculeata Miller: Uma análise bibliométrica. Brazilian Journal of Surgery and Clinical Research, 22 (3).

Moraes, T. V. de, Ferreira, J. P. G., Souza, M. R. A. de, \& Moreira, R. F. A. (2020). Atividade antioxidante e conteúdo de compostos fenólicos do chá do caule da Pereskia aculeata Miller fresco e armazenado sob congelamento. Research, Society and Development, 9(5), e34953140. https://doi.org/10.33448/rsdv9i5.3140

Moraes, J. L. A., Ferreira, P. M., Ramos, A. M. P. C., Athar, C. Y. U. P. A., Sousa, F. J. D. (2021). A utilização das redes sociais como ferramenta na 
Research, Society and Development, v. 10, n. 8, e17910817226, 2021

(CC BY 4.0) | ISSN 2525-3409 | DOI: http://dx.doi.org/10.33448/rsd-v10i8.17226

monitoria acadêmica: relato de experiência. Research, Society and Development, 10(2), e38710212360. https://dx.doi.org/10.33448/rsd-v10i2.12360

Oliveira, A. F. D. S., Khan, A. S., Lima, P. V. P. S., \& Silva, L. M. R. (2008). A Sustentabilidade da agricultura orgânica familiar dos produtores associados à APOI (Associação dos Produtores Orgânicos da Ibiapaba-CE). http://www.repositorio.ufc.br:8080/ri/handle/123456789/5495

Pinto, N. de C. C., Cassini-Vieira, P., Souza-Fagundes, E. M. de, Barcelos, L. S., Castañon, M. C. M. N., \& Scio, E. (2016). Pereskia aculeata Miller leaves accelerate excisional wound healing in mice. Journal of Ethnopharmacology, 194, 131-136. https://doi.org/10.1016/j.jep.2016.09.005

Rocha, D. R. C., Pereira Júnior, G. A., Vieira, G., Pantoja, L., Santos, A. S., \& Pinto, N. A. V. D. (2009). Macarrão adicionado de ora-pro-nóbis (Pereskia aculeata Miller ) desidratado. Alimentos e Nutrição Araraquara, 19(4), 459-465.http://servbib.fcfar.unesp.br/seer/index.php/alimentos/article/view/656

Santo Manoel, G. E. (2019). Elaboração e avaliação da qualidade sensorial e física de macarrão enriquecido com ora-pro-nóbis (Pereskia aculeata Mill). VIII Jornada de Produção Científica e Tecnológica (JPCT) e XI Ciclo de Palestras Tecnológicas (CIPATEC)-2019.

Souza, L., Caputo, L., Inchausti De Barros, I., Fratianni, F., Nazzaro, F., \& De Feo, V. (2016). Pereskia aculeata Muller (Cactaceae) Leaves: Chemical Composition and Biological Activities. International Journal of Molecular Sciences, 17(9), 1478. https://doi.org/10.3390/ijms17091478

Statista. (2021). Instagram: users by country. https://www.statista.com/statistics/578364/countries-with-most-instagram-users/

Stehmann, J. R., \& Sobral, M. (2017). Biodiversidade no Brasil. In FARMACOGNOSIA: do produto natural ao medicamento (pp. 1-10). Artmed.

Valente, C. O., Dores, G. H. S., Seifert Jr, C. A., \& Durigon, J. (2020). Popularizando as plantas alimentícias não convencionais (PANC) no sul do Brasil. Congresso Brasileiro de Agroecologia, 15(2), 1-6. http://cadernos.aba-agroecologia.org.br/index.php/cadernos/article/view/3458 